\title{
Two-dimensional ultrasound: can it replace computed tomography in liver volume assessment?
}

\author{
Shimaa Farghaly', Marwa Makboul ${ }^{1 *}$ and Mahmoud Refaat Shehata ${ }^{2}$
}

\begin{abstract}
Background: Liver volume estimation is considered as an integral part in preoperative evaluation in patients undergoing liver transplantation; computed tomography and magnetic resonance imaging are considered the gold standard methods for liver volume estimation, and both are reliable and valid in determination of liver volume via manual and semi-automated methods. Reliable and accurate set of three simple measurement planes using twodimensional ultrasound for volumetric assessment of liver was determined, and predictive equation using these three simple measurements was performed, which is simple to perform and easy to calculate, in order to evaluate liver volume and validate these measurements against CT images. Our aim in this study was to evaluate the efficacy and validity of two-dimensional ultrasound in liver volume estimation compared to CT volumetry as a gold standard.
\end{abstract}

Results: A strong linear positive correlation with no statistical significant difference was found between 2D US and semi-automated $C T$ volumetric, and result was $r=0.7402$ and $p>0.05$, with an average liver volume of $1572.10( \pm$ $326.43) \mathrm{cm}^{3}$ and $1559.30( \pm 381.02) \mathrm{cm}^{3}$ respectively.

No statistically significant difference was found also between the two modalities in different age groups and different sexes.

Conclusion: Simple linear two-dimensional ultrasound could be considered an efficient, accurate, and trustable tool for liver volume measurement in clinical practice.

Keywords: Liver, Volumetry, Two-dimensional ultrasound, Semi-automated technique, Multi-slice computed tomography

\section{Background}

Liver volume estimation is considered as an integral part in preoperative evaluation in patients undergoing liver transplantation and determines the surgical respectability of hepatic focal lesions by assessment of remnant liver volume [1].

Computed tomography (CT) is a very good tool for volume assessment as it is noninvasive with high contrast and spatial resolution [2]. Manual tracing of the liver boundaries on individual CT images is the standard technique for liver volume calculation, and this yields accurate but subjective results; however, this technique

\footnotetext{
*Correspondence: drmarwa201198@ymail.com; makboul@aun.edu.eg ${ }^{1}$ Radiodiagnosis Department, Faculty of Medicine, Assiut University, Assiut, Egypt

Full list of author information is available at the end of the article
}

is time-consuming as it takes $25-40$ min on average for liver volume assessment in one patient [3]. Automated computerized liver volumetry developed with advanced technology replaced manual liver volumetry [4]. Luciani et al. [5] reported that manual and automated CT liver volumetry were strongly correlated, but automated method was significantly time saving than the manual method.

Semi-automated segmentation techniques using mathematical models based on histogram cluster analysis were introduced [6], with time saving and improvement in accuracy and repeatability [7]. Although computed tomography (CT) and magnetic resonance imaging (MRI) are considered the gold standard methods for liver volume estimation, and both are reliable and valid in determination of liver volume via manual and semi- 
automated methods [8], CT has risk of ionizing radiation, and MRI causes issues of claustrophobia and it is contraindicated in the case of pacemaker and cochlear implants [9]. So, there is still a need for a simple, reliable, uncomplicated, and valid technique that can be trusted for liver volume assessment [10].

Ultrasound evaluation of liver size is available, inexpensive, and comparably a fast imaging technique which does not use ionizing radiation, and single, simple linear ultrasound measurements of the liver are easily performed, but they are limited, as they provide dimensional information at only one site within one lobe of the liver (right lobe) [11].

Many attempts to predict liver volume from simple linear ultrasound measurements was performed. Studies of Elstein et al., Glenn et al., and Zoli et al. [11] have previously developed equations to determine liver volume, but their accuracy was not reported. Kitajima et al. [12] reported the possibility of performing liver volumetry by means of conventional ultrasound in good correlation with actual specimen volume. Also, $\mathrm{Xu}$ et al. [13] reported the usefulness of three-dimensional ultrasound (3D US) volumetric evaluation in correlation with true volume; however, it is not universally proven to be successful for 3D evaluation of the liver because of its limitations, both physically and those related to variable reproducibility of the exam.

Childs et al. [10] determined reliable and accurate set of three simple measurements planes using twodimensional (2D) ultrasound for volumetric assessment of liver .Then, Childs et al. [14] performed predictive equation using these three simple measurements, which is simple to perform and easy to calculate, in order to evaluate liver volume and validate these measurements against CT images.

The aim of this study is to evaluate the efficacy and validity of $2 \mathrm{D}$ ultrasound in liver volume estimation using Childs et al.'s equation compared to CT volumetry as a gold standard.

\section{Methods}

2D ultrasound volumetric assessment of the liver was performed in 100 patients who underwent tri-phasic multi-slice computed tomography (MSCT) abdomen for reasons other than related to liver such as acute abdomen, renal mass assessment, adnexal, and pelvic lesions which were enrolled in this prospective study from October 2017 to March 2018. Patients under 18 years old or with abnormal liver function tests were excluded.

The study was carried out after obtaining the permission of the Ethics Committee of Scientific Research, with informed consent from the patients.

All CT examination was performed using 16-MSCT scanner (Light speed, GE Healthcare, USA) with tube voltage of $120 \mathrm{KV}$, tube current of $150-280 \mathrm{~mA}$, and a $512 \times 512$-pixel matrix. Then, data were reconstructed into 0.625 -mm-slice images.

Non-contrast standard acquisition of abdomen was done first, followed by triphasic examination by administration of $100 \mathrm{ml}$ of non-ionic contrast agent Ultravist $370 \mathrm{mg}$ iodine concentration (Iopromide $0.769 \mathrm{~g}$ ) in arterial, portal, and delayed phases. Then, volumetric assessment of the liver was done using semi-automated segmentation technique on Advantage Adw 4.6 GE work station.

\section{Data analysis}

CT liver volumes were calculated by two radiologists conjoint with more than 10 years' experience in CT using liver analysis application software, which used the density differences in portal/venous scan images to obtain semi-automated liver segmentation. The gall bladder and the main vessels, such as the retro-hepatic inferior vena cava vein, were manually excluded from segmentation volume. A volumetric reconstruction of the liver and quantification of total liver volume (TLV) were obtained.

The semi-automated volumetric measurement was divided into three steps (Fig. 1). The first step was manual delineation, which worked on two-dimensional (2D) view. It captured the cross section of the liver by user input control points. The second step was 3D surface reconstruction which worked with radial basis function based on the manual delineation. The last step was surface evolution, which evolved the reconstructed 3D surface using a level-set framework comprising both image and shape information.

\section{Ultrasound volumetry}

Two radiologists with more than 10 years' experience in US performed U/S examination conjointly using Philips ultrasound system (Affiniti 50G, Germany) with convex probe C6-2.

\section{Two images of the liver were taken according to Childs et al.}

- The first image (termed image 1) was taken with the transducer placed longitudinally in the midline parallel to the xiphisternum for left hepatic lobe delineation.

- The second image (termed image 2) was taken with the transducer orientated longitudinally in the midclavicular line for right hepatic lobe delineation.

One measurement was taken from image 1, a linear measurement of the maximum anterior to posterior diameter of the liver (measurement A), as shown in Fig. 2a, and two measurements were taken from image 2 , a linear 

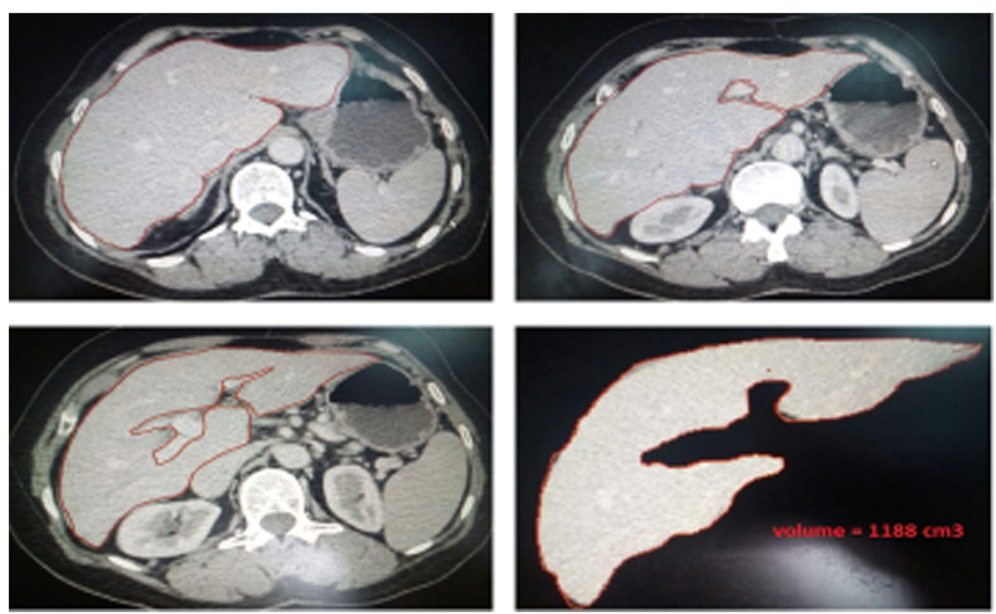

Fig. 1 Example of semi-automated CT volumetry steps

measurement from the dome to the tip of the right lobe of the liver (measurement B) as shown in Fig. 2b and a linear measurement of the maximum anterior to posterior diameter of the liver in this image (measurement $\mathrm{C}$ ) as shown in Fig. 2c.

The three measurements were then used to determine liver volume according to Childs et al.'s equation [14] as liver volume $\left(\mathrm{cm}^{3}\right)=343.71+(0.84 \times \mathrm{ABC})$ where $\mathrm{ABC}$ is the product of the three linear measurements.

Data were statistically described in terms of range, mean, standard deviation, frequencies (number of cases), and percentages when appropriate.

Difference between variables was done using MannWhitney test. Statistical significance was set at $p<0.05$, and all reported $p$ values were two sided. Correlation coefficient $(r)$ between two variables was set as value of $r$ which is always between +1 and -1 .

\section{Results}

One hundred patients were included in this study, 50 males and 50 females, with age ranged from $28-77$ years, and the mean age was 50.58 years.
The liver volume was estimated for each case by both $\mathrm{U} / \mathrm{S}$ and semi-automated MSCT. The average liver volume by U/S was $1572.10 \pm 326.43 \mathrm{~cm}^{3}$, while the average liver volume by semi-automated CT was $1559.30 \pm$ $381.02 \mathrm{~cm}^{3}$ (Table 1, Fig. 3), with no statistical significant difference found between both of them as the $p$ value was 0.798 (>0.05).

As regards age, average liver volume obtained by U/S in cases less than 50 years was $1524.80 \pm 316 \mathrm{~cm}^{3}$ and in more than 50 years was $1612.40 \pm 332.38 \mathrm{~cm}^{3}$, while the average liver volume by $\mathrm{CT}$ in cases less than 50 years was $1504.90 \pm 337.27 \mathrm{~cm}^{3}$ and in more than 50 years was $1605 \pm 412.14 \mathrm{~cm}^{3}$, with no statistical significant difference found in both methods as the $p$ value was 0.182 and 0.19 respectively $(>0.05)$ (Table 2 ).

The average liver volume by U/S in male was 1606.10 $\pm 398.04 \mathrm{~cm}^{3}$, while in female was $1538.10 \pm 233.42$ $\mathrm{cm}^{3}$, and the average liver volume in male by semiautomated CT volumetry was $1566 \pm 418.22 \mathrm{~cm}^{3}$, while in female was $1552.50 \pm 343.98 \mathrm{~cm}^{3}$, with also no statistical significant difference found between them as regards sex as the $p$ value was 0.3 and 0.86 respectively $(>0.05)$ (Table 3).
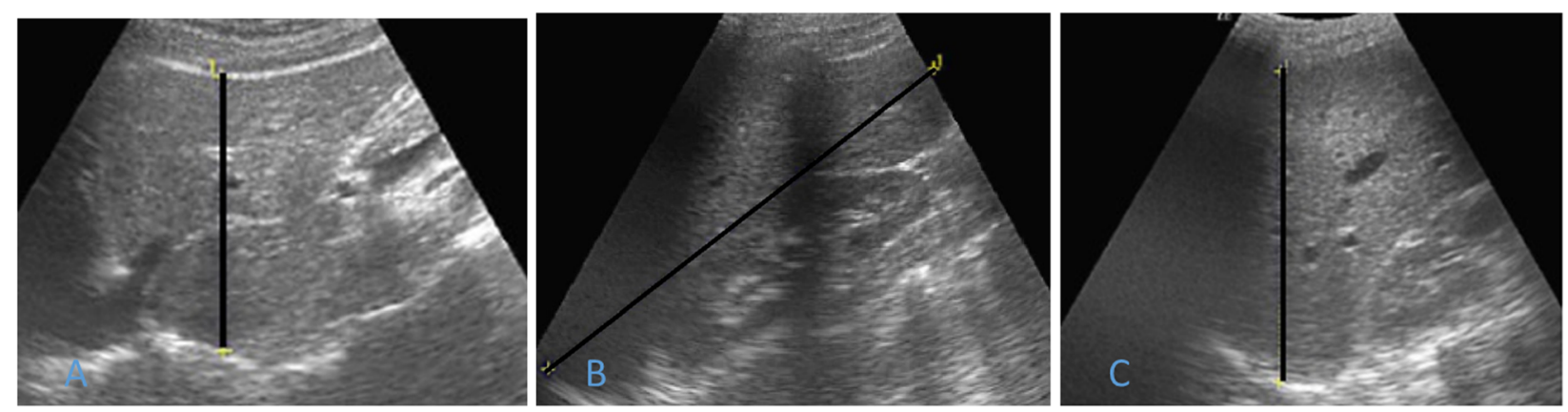

Fig. 2 The three linear measurements of liver. a The left lobe of the liver. b, c The right lobe of the liver 
Table 1 Comparison of average liver volume by US and semiautomated CT volumetry

\begin{tabular}{lll}
\hline Method & Mean \pm SD $(N=100)$ & $p$ value \\
\hline US & $1572.10 \pm 326.43$ & 0.798 \\
MSCT & $1559.30 \pm 381.02$ & \\
\hline
\end{tabular}

In the evaluation of linear association with the Pearson product-moment correlation coefficient $(r)$ (which measures the strength and direction of the linear relationship between two variables on a scatter plot) by comparing the results obtained by U/S volumetry with semiautomated MSCT volumetry as gold standard, we found that the value of $r$ was 0.7402 which means strong linear positive correlation between them (Fig. 4).

\section{Discussion}

Liver size is a significant indicator for the presence of a disease process; also, it aids in treatment planning for liver transplantation and resection. In addition, it is used post-therapy for assessment of graft regeneration and treatment response evaluation in case of liver malignancy [1], and it is used after portal vein embolization for evaluation of the increase in future liver remnant volume $[15,16]$.

Liver volume is a better way for the assessment of liver size as it represents the entire liver rather than linear measurements in single planes [17]. Computed tomography $(\mathrm{CT})$ has been widely used for volumetric assessment of the liver, magnetic resonance imaging (MRI)
Table 2 Average liver volume by US and CT as regards age

\begin{tabular}{llll}
\hline & Age & & \\
\cline { 2 - 4 } & Less than $50(N=46)$ & 50 or more $(N=54)$ & $p$ value \\
\hline US & $1524.80 \pm 316.34$ & $1612.40 \pm 332.38$ & 0.182 \\
MSCT & $1504.90 \pm 337.27$ & $1605.50 \pm 412.14$ & 0.19 \\
\hline
\end{tabular}

and ultrasound also, and has shown trustable organ volume measurements in light of appropriate scanning protocols [1].

Many previous studies have tried to evaluate and validate various imaging modalities for liver volumetry; however, there is no clear evidence about the most accurate method [5].

CT volumetry in combination with dedicated software plays important role in the evaluation of candidates for liver donor transplantation as it shows accurate estimation of graft dimension before transplant [18]. It has been performed by manual tracing of the liver boundary and summation of the liver area on each axial section, but this technique is operator dependent and timeconsuming.

Although with advanced technology, automated and semi-automated volumetric measurements have been carried out to replace manual liver volumetry for accurate liver volume calculation [3]; however, there is still need for simple and uncomplicated technique that can be trusted for liver volume assessment [10].

Despite the widespread 3D ultrasound and its simplicity in the assessment of liver size, it is not proved to be
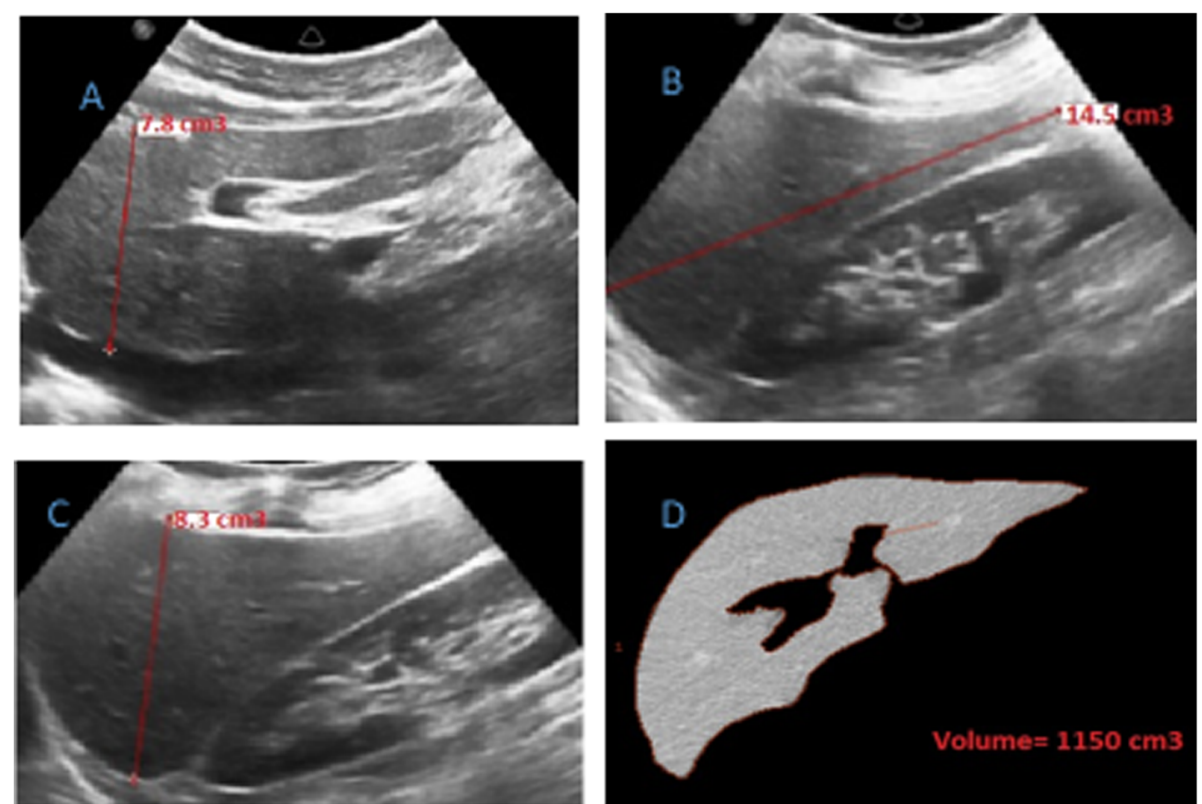

Fig. 3 Female patient, 50 years old. a Image 1 represents left lobe of the liver $(7.8 \mathrm{~cm})$. b Image 2 represents right lobe of the liver $(14.5 \mathrm{~cm})$. c Image 3 represents right lobe of the liver $(8.3 \mathrm{~cm})$. Liver volume $(\mathrm{cm} 3)=343.71+(0.84 \times A B C)=343.71+(0.84 \times 7.8 \times 14.5 \times 8.3)=1132.2 \mathrm{~cm}^{3}$. d Semi-automated CT volumetry for the same case with liver volume was $1150 \mathrm{~cm}^{3}$ 
Table 3 Average liver volume by US and CT volumetry as regards sex

\begin{tabular}{llll}
\hline & Sex & & \\
\cline { 2 - 4 } & Male $(N=50)$ & Female $(N=50)$ & $p$ value \\
\hline US & $1606.10 \pm 398.04$ & $1538.10 \pm 233.42$ & 0.3 \\
MSCT & $1566.00 \pm 418.22$ & $1552.50 \pm 343.98$ & 0.86 \\
\hline
\end{tabular}

a successful method for liver volumetric assessment because of its limitations, both physically as it is impractical and time-consuming and also those related to a variable reproducibility of the examination which depends mainly on the examiner skills [19]. Also, in the assessment of liver size by simple linear 2D US, there is a lack of standardization of measurement method and adopted cutoff values with the accepted plane of measurement, and its used cutoff values varies from one department to the other, so most of the previous studies did not report its accuracy [10, 17, 20, 21].

As the single linear liver size measurement by ultrasound represents the liver in only one plane, and the morphologic shape of the liver differs among people, Childs et al. determined the set of practical three simple measurement planes using 2D US, and predictive equation was created from these three measurements to determine the liver volume which is more accurate than the liver size [11].
In this study, the 2D US volumetric assessment of the liver in different age groups and different sexes was done by using Childs et al.'s [14] equation, and the results were compared with semi-automated CT volumetric results as a gold standard.

To our knowledge, Childs et al.'s study [19] is the only study which is comparing the results of $2 \mathrm{D}$ US volumetric assessment of the liver based on Child et al.'s [14] equation with CT volumetry and reported near perfect agreement $(r=0.9)$ between the two modalities [11, 19]. This agreed with our study which also reported a strong positive correlation $(r=0.74)$ with no statistically significant difference found between them $(p>0.05)$, and no statistically significant difference was found also between them in different age groups and different sexes.

A limitation of this study was that this study was conducted only on normal liver cases, so, in order to generalize the results, further future studies are recommended on diseased liver to validate its accuracy in liver volumetric assessment.

\section{Conclusion}

In conclusion, we found that simple linear $2 \mathrm{D} \mathrm{U} / \mathrm{S}$ could be an efficient, accurate, and trustable method for liver volume measurement in clinical practice.

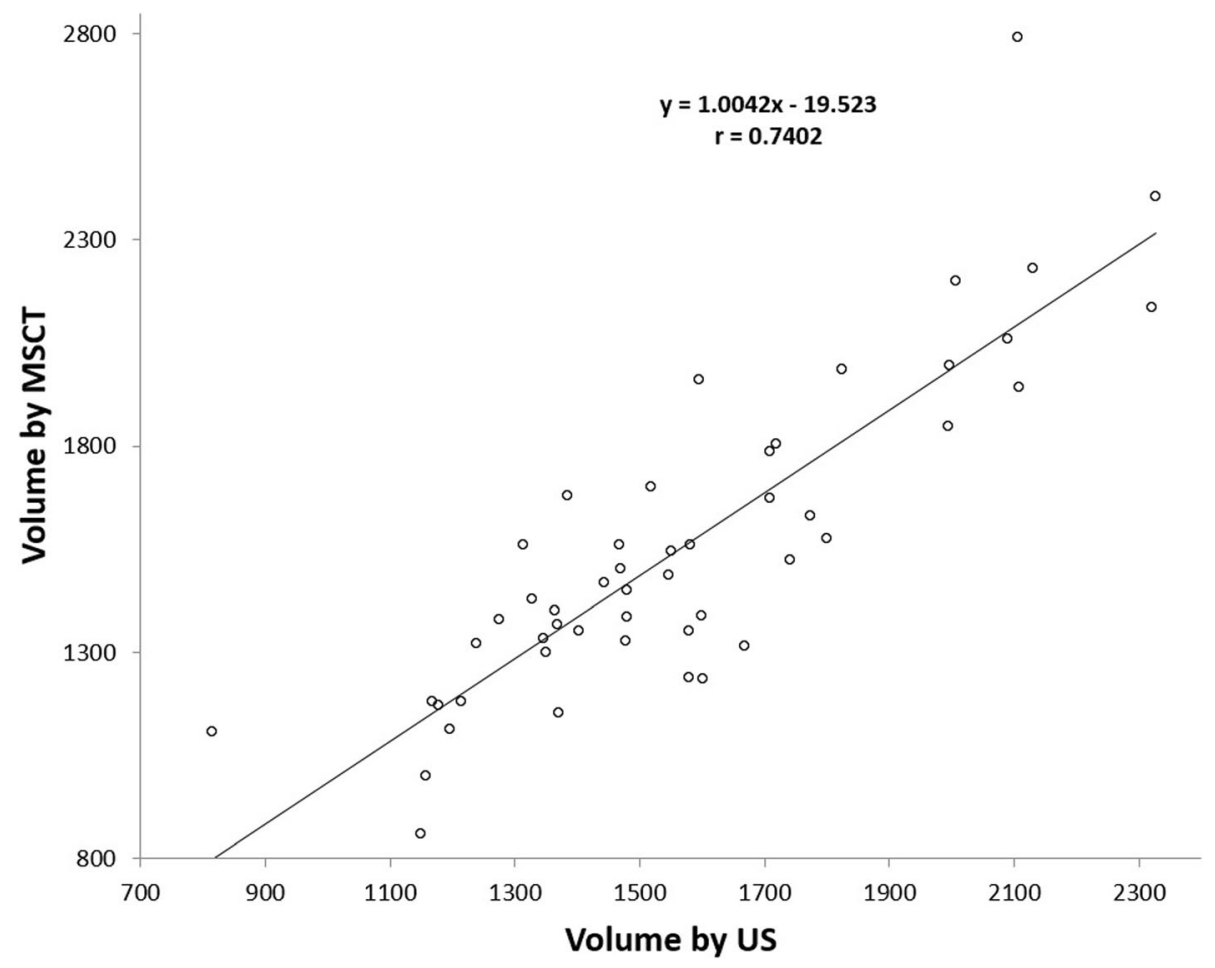

Fig. 4 Scatter plot diagram is showing correlation coefficient between US and semi-automated CT volumetry with strong positive correlation 


\section{Abbreviations}

2D US: Two-dimensional ultrasound; 3D US: Three-dimensional ultrasound; $\mathrm{CT}$ : Computed tomography; MRI: Magnetic resonance imaging; TLV: Total liver volume

\section{Acknowledgements}

Not applicable.

\section{Authors' contributions}

With the submission of this manuscript, I would like to declare that all authors have contributed sufficiently to the scientific work regarding study concepts and design, clinical studies, collection of the data, statistical analysis, manuscript preparation, and editing. All authors have read and approved the manuscript.

\section{Funding}

This study had no funding from any resource.

\section{Availability of data and materials}

The datasets used or analyzed during the current study are available from the corresponding author on reasonable request.

\section{Ethics approval and consent to participate}

This study was approved by the research ethics committee of the Faculty of Medicine at Assiut University in Egypt on 2018, reference number (17300313), and all patients included in this study gave written informed consent to participate in this research.

\section{Consent for publication}

All patients included in this research gave written informed consent to publish the data contained within this study.

\section{Competing interests}

The authors declare that they have no competing interests.

\section{Author details}

${ }^{1}$ Radiodiagnosis Department, Faculty of Medicine, Assiut University, Assiut, Egypt. ${ }^{2}$ General Surgery Department, Faculty of Medicine, Assiut University, Assiut, Egypt.

Received: 26 September 2019 Accepted: 29 October 2019

Published online: 10 December 2019

\section{References}

1. Lim MC et al (2014) CT volumetry of the liver: where does it stand in clinical practice? Clin Radiol 69(9):887-895

2. Lemke AJ et al (2006) Living donor right liver lobes: preoperative CT volumetric measurement for calculation of intraoperative weight and volume. Radiology 240(3):736-742

3. Nakayama $Y$ et al (2006) Automated hepatic volumetry for living related liver transplantation at multisection CT. Radiology 240(3):743-748

4. Bae KT et al (1993) Automatic segmentation of liver structure in CT images. Med Phys 20(1):71-78

5. D'Onofrio M et al (2014) Liver volumetry: is imaging reliable? Personal experience and review of the literature. World J Radiol 6(4):62-71

6. Gao L et al (1996) Automatic liver segmentation technique for threedimensional visualization of CT data. Radiology 201(2):359-364

7. Suzuki K et al (2011) Quantitative radiology: automated CT liver volumetry compared with interactive volumetry and manual volumetry. AJR Am J Roentgenol 197(4):W706-W712

8. Shores NJ et al (2011) Non-contrasted computed tomography for the accurate measurement of liver steatosis in obese patients. Dig Dis Sci 56(7): 2145-2151

9. Dill T (2008) Contraindications to magnetic resonance imaging: non-invasive imaging. Heart 94(7):943-948

10. Childs JT, Esterman AJ, Thoirs KA (2014) Ultrasound measurements of the liver: an intra and inter-rater reliability study. Australas J Ultrasound Med 17(3):113-119

11. Jessie T, Child KAT, Esterman AJ (2016) the development of a practical and uncomplicated predictive equation to determine liver volime from simple linear ultrasound measurments of the liver. Radiography 22:e(125)-e(130)
12. Kitajima K et al (2008) Sonographic preoperative assessment of liver volume before major liver resection. Gastroenterol Clin Biol 32(4):382-389

13. Xu HX et al (2003) Estimation of liver tumor volume using a three-dimensional ultrasound volumetric system. Ultrasound Med Biol 29(6):839-846

14. Childs JT, Thoirs KA, Esterman AJ (2016) The development of a practical and uncomplicated predictive equation to determine liver volume from simple linear ultrasound measurements of the liver. Radiography 22(2):e125-e130

15. Suzuki K et al (2010) Computer-aided measurement of liver volumes in CT by means of geodesic active contour segmentation coupled with levelset algorithms. Med Phys 37(5):2159-2166

16. Yamanaka J, Saito S, Fujimoto J (2007) Impact of preoperative planning using virtual segmental volumetry on liver resection for hepatocellular carcinoma. World J Surg 31(6):1249-1255

17. Kratzer W et al (2003) Factors affecting liver size: a sonographic survey of 2080 subjects. J Ultrasound Med 22(11):1155-1161

18. Ringe Kl et al (2012) Evaluation of living liver donors using contrast enhanced multidetector $C T$ - The radiologists impact on donor selection. BMC Med Imaging 12:21

19. Oldhafer KJ et al (1999) 3-dimensional (3-D) visualization of the liver for planning extensive liver resections. Chirurg 70(3):233-238

20. Gosink BB, Leymaster CE (1981) Ultrasonic determination of hepatomegaly. J Clin Ultrasound 9(1):37-44

21. Niederau C et al (1983) Sonographic measurements of the normal liver, spleen, pancreas, and portal vein. Radiology 149(2):537-540

\section{Publisher's Note}

Springer Nature remains neutral with regard to jurisdictional claims in published maps and institutional affiliations.

\section{Submit your manuscript to a SpringerOpen ${ }^{\circ}$ journal and benefit from:}

- Convenient online submission

- Rigorous peer review

- Open access: articles freely available online

- High visibility within the field

- Retaining the copyright to your article

Submit your next manuscript at $\boldsymbol{\nabla}$ springeropen.com 